\title{
Shear-banding and microstructure of colloids in shear flow
}

\author{
Jan K. G. Dhont, ${ }^{* a}$ M. Pavlik Lettinga, ${ }^{a}$ Zvonimir Dogic, ${ }^{a}$ Tjerk A. J. Lenstra, ${ }^{a}$ \\ Hao Wang, ${ }^{a}$ Silke Rathgeber, ${ }^{a}$ Philippe Carletto, ${ }^{a}$ Lutz Willner, \\ Henrich Frielinghaus ${ }^{b}$ and Peter Lindner ${ }^{c}$
}

${ }^{a}$ Forschungszentrum Jülich, IFF/Weiche Materie, D-52425, Jülich, Germany
${ }^{b}$ Forschungszentrum Jülich, IFF/Neutronen Streuung, D-52425, Jülich, Germany
${ }^{c}$ Institute Laue-Langevin, 6 rue Jules Horowitz, F-38042, Grenoble, cedex 9, France

Received 23rd May 2002, Accepted 7th June 2002

First published as an Advance Article on the web 20th September 2002

\begin{abstract}
We shall discuss the following phenomena found in various colloidal systems in shear flow. We recently observed shear-banding in suspensions of fd-virus in a cylindrical shear cell. Small angle light scattering experiments revealed that the shear-banding transition is preceded by a relatively fast process (minutes) of nematic-to-paranematic phase separation during which inhomogeneities on the micrometer length scale are formed. After the formation of these inhomogeneities, a slow (hours) appearence of shear-bands is observed, which have a height of a few $\mathrm{mm}$. In the stationary state it is found, by means of polarization microscopy, that inhomogeneities exist within the bands. Small angle, time resolved light scattering experiments on near-critical microstructural order in a mixture of colloidal spheres and free polymer under stationary shear flow are discussed. The unexpected distortion of microstructure in directions perpendicular to the flow direction is quantitatively explained by extending an already existing theory, to include shear-induced short-ranged microstructural distortion. In colloidal systems consisting of "hairy colloids", where a spherical core is decorated with relatively long polymers, shear-induced polymer brush deformation might be important for its structural and rheological behavior. Preliminary neutron scattering and rheology experiments are performed to study polymer brush deformation in (semi-) dilute suspensions.
\end{abstract}

\section{Introduction}

In this Faraday Discussion contribution we shall discuss three subjects that are currently of interest in the group "Weiche Materie" (German for "Soft Matter") at the Jülich Research Center:

(i) Shear-banding in suspensions of rods,

(ii) Shear-induced microstructure in near-critical colloids,

(iii) Very soft, "hairy" colloids in shear flow.

Results that are presented here are partly of a preliminary nature. Each of the following sections contains an introduction to the subject at hand.

\section{Shear-banding in suspensions of fd-virus}

Shear-banding is the phenomenon where macroscopically large regions (the "bands"), with differing microstructural properties, coexist under the influence of shear flow. Banded structures are 
found at very low shear-rates, far below the shear-rate where the Taylor instability occurs. The final stationary state often depends on whether the flow is applied under controlled stress or shear-rate conditions. ${ }^{1,2}$ In a cylindrical geometry, bands are either alternately stacked in the vorticity direction or layered around the inner cylinder in the gradient direction (as sketched on the right in the schematic phase diagram in Fig. 1, which will be discussed in more detail below). Well studied systems that exhibit this complex behavior are dispersions of wormlike micelles under shear. Depending on the concentration of monomers and flow conditions, a variety of phase transitions and structural changes are observed. ${ }^{1,3,4,5}$ These experiments are theoretically described by Cates. ${ }^{6}$ Wormlike micelles have significant chain flexibility and they permanently exchange material (i.e. they can break and recombine). This complicates the physics of shear-banding considerably.

In order to gain fundamental insight into the origin of the shear-banding transition, we performed experiments on a much simpler system : fd-virus suspensions. Fd-virus is a good model system of hard rods ${ }^{7}$ and displays an isotropic-nematic (I-N) phase transition. The location of phase transition lines is shifted to lower concentration on applying shear flow, since shear flow stabilizes nematic order. The shear dependent location of I-N spinodals of fd-virus suspensions has been determined in ref. 8 by means of time resolved birefringence measurements. Due to very slow nucleation rates it has not been possible to determine the shear-rate dependent location of binodals and shear-banding transition lines. In the present work we added polymer to the fd-virus suspension, which widens the biphasic region, resulting in increased nucleation rates ${ }^{9}$ and shearbanding transition rates.

Fd-virus at $100 \mathrm{mM}$ ionic strength was prepared in the biphasic $\mathrm{I}-\mathrm{N}$ region without shear flow $\left(21 \mathrm{mg} \mathrm{ml}^{-1}\right)$. To this suspension, a stock solution $(30 \%)$ of dextran $513 \mathrm{kMW}$ (Sigma) was added, until the dispersion became opaque. The behaviour of this dispersion under shear flow is studied using an optical, cylindrical shear-cell, which is positioned between two polarizers in order to probe local orientational order. This shear cell allows for time resolved, small angle light scattering experiments in the flow-vorticity plane, where scattered light from one gap is blocked by means of a pinhole that is positioned in the middle of the inner cylinder. Formation of inhomogeneities on the micrometer scale were studied both by small angle light scattering (SALS) and microscopy.

We observed that the nematic phase is stable for shear-rates larger than a critical binodal shearrate $\dot{\gamma}_{\text {bin }} \approx 1.5 \mathrm{~s}^{-1}$, even though the sample without shear flow is in the I-N biphasic region. Such a critical shear-rate is predicted by a Doi-Edwards like theory and is confirmed by means of birefringence experiments. ${ }^{8}$ For shear-rates larger than $\dot{\gamma}_{\text {bin }}$ the sample hardly scatters light. When a quench is made from the stable nematic phase at high shear-rates into the biphasic region by suddenly lowering the shear-rate below the binodal shear-rate (i.e. $\dot{\gamma}<\dot{\gamma}_{\text {bin }}$ ), phase separation occurs, the kinetics of which can be followed with SALS. In Fig. 2 the evolution of the scattering pattern for two different quenches is shown. In the initial stage of phase separation, the scattering
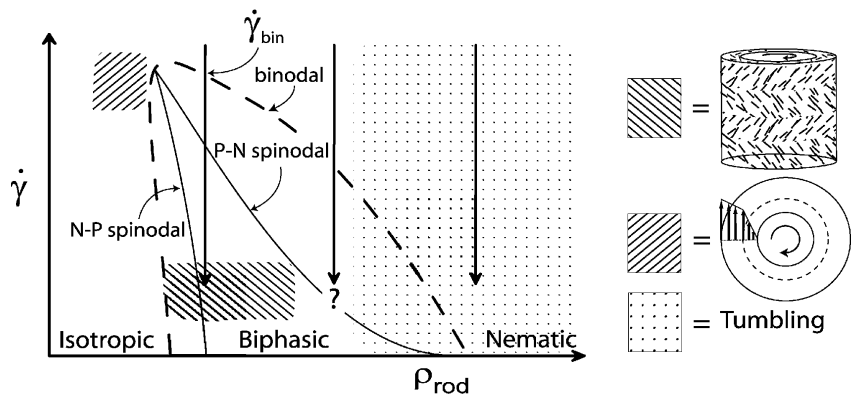

Fig. 1 Sketch of the possible phase diagram of fd-virus under shear. The vertical arrows indicate a quench of the shear-rate. The dashed line indicates the binodal. At low rod concentration in the biphasic region we observe shear-banding along the vorticity direction while at higher rod concentrations there are strong indications of rod tumbling. Shear-banding along the gradient direction is expected to occur close to the non-equilibrium critical point. The two types of stationary shear-banded states in a cylindrical shear cell are depicted on the right hand-side. The region where the sample exhibits shear-banding along the vorticity direction is completely contained within the two phase region. 


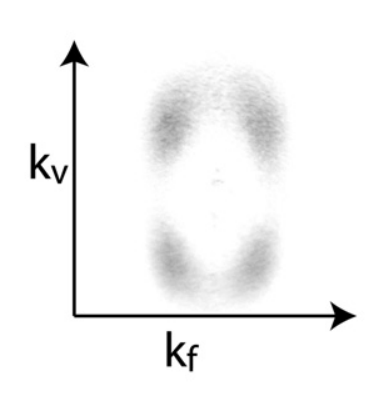

$$
\dot{\gamma}_{\text {final }}=0
$$

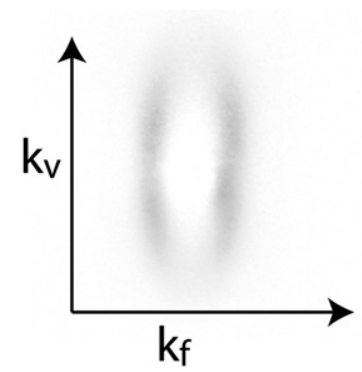

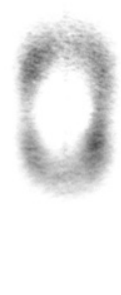

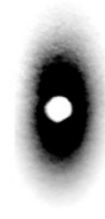

$$
\dot{\gamma}_{\text {final }}=0.3 \mathrm{~s}^{-1}
$$

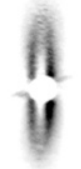

Fig. 2 SALS images taken between $5 \mathrm{~s}$ and 5 min after a shear-rate quench from $\dot{\gamma}=2 \mathrm{~s}^{-1}$ to $\dot{\gamma}=0 \mathrm{~s}^{-1}$ (top) and $\dot{\gamma}=0.3 \mathrm{~s}^{-1}$ (bottom). The first images on the left are taken a few seconds after the quench, while the images on the right are taken after about three minutes. $k_{\mathrm{v}}$ and $k_{\mathrm{f}}$ indicate the vorticity and flow direction, respectively.

pattern shows four lobes, which indicates the formation of micron-sized paranematic regions within the nematic, which regions are tilted with respect to the flow direction (a paranematic state is a shear-aligned, otherwise isotropic state). After a few minutes, an intermediate state exists in which these regions are aligned along the flow direction.

In the shear-rate regime $\dot{\gamma}=0.1-0.4 \mathrm{~s}^{-1}$, in addition to the fast phase separation described above (few minutes) an additional process of band formation is observed on much longer time scales (few hours), as is shown in Fig. 3. These bands are alternately stacked in the vorticity direction and can only be detected under crossed polarizers. The bands can be as broad as $5 \mathrm{~mm}$,
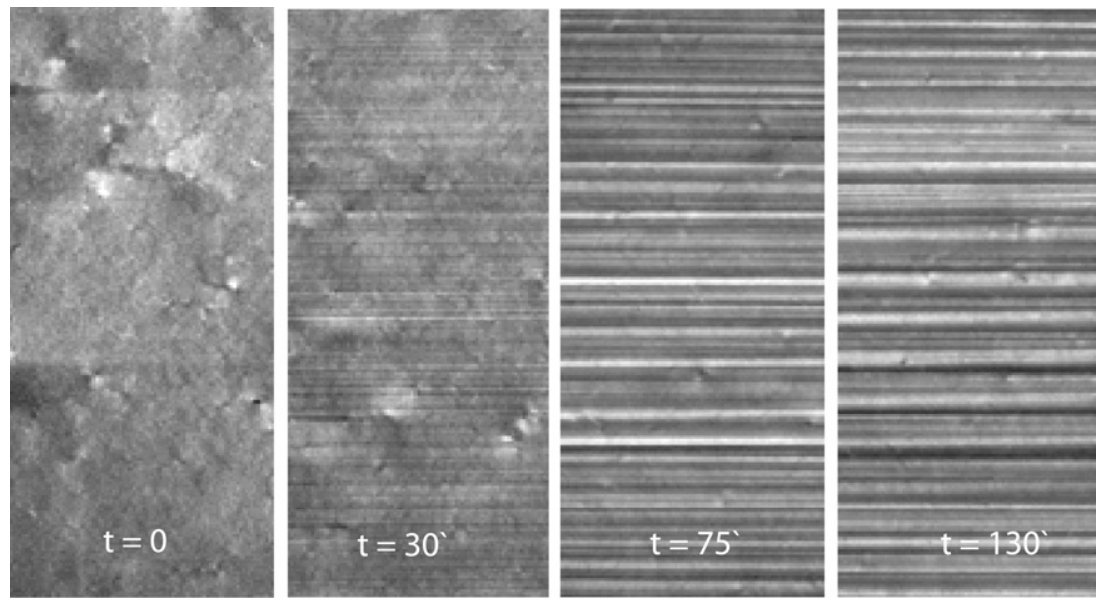

Fig. 3 CCD-images from the side of the cylindrical shear-cell (width $4 \mathrm{~cm}$ ) during band formation over a time span of $2 \mathrm{~h}$. The shear-cell is positioned between two crossed polarizers. The shear-rate was $\dot{\gamma}=0.3 \mathrm{~s}^{-1}$. 


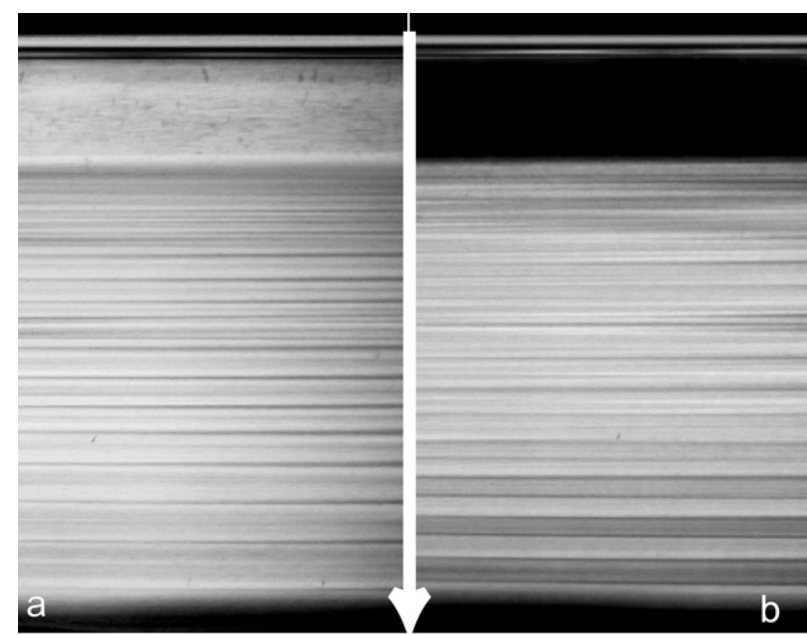

Fig. 4 (a) Shear-bands of a sample that has been sheared for a week. During this time some sedimentation of the more dense nematic regions takes place. (b) Same as figure (a), but immediately after the cessation of shear flow. In this sample, bands fully disappear over a period of an hour.

depending on the composition of the sample. Bands are formed within a limited virus concentration range. The lower concentration at which banding still occurs is set by the lower branch of the binodal. For samples that are outside the two-phase region, no shear-banding along the vorticity direction is observed. This is illustrated in Fig. 4a, where an image is shown of a sample that has been sheared for a week. During this time a gradient in density is established due to density differences of coexisting paranematic and nematic phases. The top part of the sample has a concentration that is equal to the lower binodal concentration. This part of the sample is a uniform paranematic phase that shows no banded flow and appears uniformly birefringent due to shear alignment. After cessation of shear flow, this paranematic phase almost instantaneously becomes isotropic, that is, the birefringence disappears and the corresponding part of the image turns black, as can be seen in Fig. 4b. Note that the size of the bands increases towards the bottom of the shear cell where the concentration of the rods is higher. Preliminary rheology experiments indicate that the high concentration limit for the occurrence of banded flow is set by the concentration where tumbling is observed right after a shear-rate quench. Here, a few damped oscillations in the stress response are observed after an instantaneous change of the shear-rate. It seems that tumbling sets in at a concentration where shear-band formation is not observed anymore, that is, tumbling probably destroys the shear-banding instability. This is in disagreement with suggestions made in refs. 10 and 11, where sign changes of normal stress differences are held responsible for the origin of the shear-banding instability, which in turn were shown to originate from tumbling domains.

A striking feature of the shear-bands is that they have an inhomogeneous internal structure, as is shown in the micrograph in Fig. 5. This type of internal structure already exists during the process of band formation, and is probably reminiscent of the micron sized structures that are formed right after the quench as seen by SALS. This is in contrast to micellar systems, where the bands of either low or high viscosity appear homogeneous.

On the basis of these preliminary results, a schematic non-equilibrium phase diagram in the shear-rate versus concentration diagram is constructed in Fig. 1. This phase diagram should be compared to the theoretical diagrams obtained by Olmsted and co-workers. ${ }^{12,13}$ The P-to- $\mathrm{N}$ and Nto-P spinodals and the binodal, also shown in this diagram, meet in a "non-equilibrium critical point", above which no phase separation occurs (in ref. 8 experiments on the shear-rate dependent location of spinodals are reported). The hatched area at low shear-rates within the biphasic region is the area where the above described shear-banding along the vorticity direction occurs. This shear-banding region in the phase diagram ends just before, precisely at, or just overlaps with the region where tumbling is observed (as indicated by the middle arrow with the question mark). 


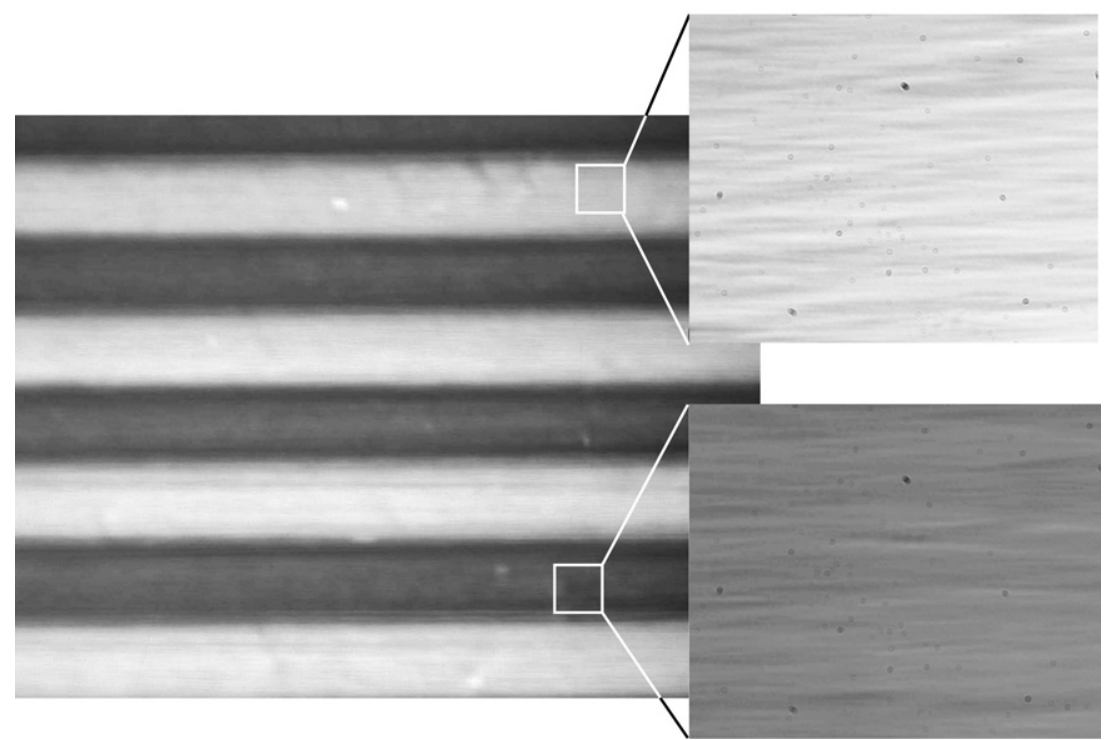

Fig. 5 The final, shear-band state, with two close-ups taken with a home-made polarization microscope. The shear-bands are about $2 \mathrm{~mm}$ in height. The typical height of the elongated inhomogeneities within the bands is about 10 to $20 \mu \mathrm{m}$.

In the hatched area close to the non-equilibrium critical point, shear-banding along the gradient direction is theoretically expected. The stable state is now a flow profile that is sketched in Fig. 1 as a top view of the cell, where a region close to the rotating inner cylinder with a high shear-rate coexists with a region with small shear-rate close to the stationary outer cylinder. For very small gap widths, the shear-rate is virtually constant within the "bands". However, the range of concentration where banding is expected is extremely small and the difference in shear-rate in the two bands is also very small. This type of shear-banding is probably experimentally of no relevance.

In conclusion, we have presented preliminary studies of the behavior of rods with attractive interactions in shear flow. In the process of shear-banding, where bands are formed along the vorticity direction, there is a fast process (minutes) where micron-sized inhomogeneities are formed, as measured by SALS, after which bands are formed on a very much longer time scale (hours), as seen through crossed polarizers. Banding is only observed in the two-phase region at concentrations below the concentration where tumbling is found. The goal of our further research will be to measure the entire non-equilibrium phase diagram as sketched in Fig. 1 and to understand the banding transition on a microscopic level. An open question is what properties of normal stress differences are responsible for the shear-banding instability. The suggestion in refs. 10 and 11, where sign changes of normal stress differences are held responsible for the origin of the shearbanding instability, which in turn were shown to originate from tumbling domains, is in contradiction with our findings: shear-banding ceases to occur where tumbling sets in. It might be that the micron-sized inhomogeneities that are formed due to phase separation are responsible for the occurrence of banding. If this is indeed the case, a linear stability analysis of appropriate equations of motion will not reveal the true nature of this instability. Equations of motion which include possibly very large gradients in suspension properties should be derived (submitted), and must be solved numerically in order to construct the phase diagram.

\section{Shear-induced distortion of the microstructure in near-critical colloids}

In this section we discuss small angle light scattering by a near-critical colloidal system under shear flow. Since an earlier, similar study, ${ }^{14}$ we have now built an optical shear cell that allows for quantitative measurements, which can be compared in detail to theoretical predictions. Earlier 
quantitative studies on shear-induced phenomena close to the gas-liquid critical point relate to integrals over the structure factor, such as the critical divergence of the shear-viscosity, ${ }^{15}$ the nonanalytic dependence of turbidity on shear-rate ${ }^{16}$ and the critical behavior of flow induced dichroism. ${ }^{17}$ These integrated quantities were shown to agree with a theory for the structure factor distortion of near-critical colloids ${ }^{18,19}$ (except for the shear-induced dichroism on close approach of the critical point). The newly developed optical shear cell allows for accurate small angle scattering measurements under shear flow, and thus enables one to directly compare the experimental structure factor itself (and not just integrated quantities) with the theory developed earlier in refs. 18 and 19. Contrary to the theoretical prediction, we find experimentally a significant distortion of the structure factor at small scattering angles in directions perpendicular to the flow direction. It will be shown that shear-induced distortion of short-ranged correlations (which were neglected in an earlier theory developed in refs. 18 and 19) are responsible for the observed distortion of longranged correlations perpendicular to the flow direction. The importance of distortions on small scale for the distortion on large scale critical microstructure partly destroys universality as it is known for the critical behaviour of systems in the absence of shear flow, in the sense that critical microstructure under shear flow depends on short-ranged correlations, and thereby on the details of the pair-interaction potential. The integrated quantities mentioned above are not very sensitive to distortions in directions perpendicular to the flow direction, since a finite distortion is found only within a limited region in wavevector space at small wavevectors, where the corresponding integrands are relatively small.

In the following we first discuss the above mentioned theoretical extension, present experiments for stationary shear flow, and analyze the data in terms of the earlier and the extended theory.

We are currently analyzing similar measurements under oscillatory shear flow. These data will be published in the near future.

\subsection{The role of shear-induced distortion of short-ranged correlations}

In the derivation of an equation of motion for the shear-rate dependent, total-correlation function $h(\boldsymbol{r})$ in refs. 18 and 19 from the $N$-particle Smoluchowski equation, the following steps are made.

(i) The three-particle correlation function is approximated by a modified Kirkwood factorization, as proposed by Fixman in ref. 20.

(ii) The equation of motion is linearized with respect to $h(r)$ for $r \gg R_{V}$, with $R_{V}$ the range of the pair-interaction potential.

(iii) Hydrodynamic interactions between the colloidal particles are neglected.

(iv) For distances $r \leqslant R_{V}$, the pair-correlation function $g=h+1$ is taken equal to the equilibrium correlation function (in the absence of shear flow).

The second step leads to an equation of motion for the asymptotic form of the total-correlation function for large distances (since $h(\boldsymbol{r}) \rightarrow 0$ as $r \rightarrow \infty$ ), which determines the structure factor at small scattering angles. In the absence of shear flow the resulting structure factor is just the Ornstein-Zernike structure factor. As will be argued later in this subsection, non-linear terms in $h$ must be taken into account on very close approach of the critical point. The validity of the neglect of hydrodynamic interactions has been verified by including hydrodynamic interactions on the Oseen level (which would be the most important contribution for the large scale structures of interest here), using parameters that apply to our experimental system (for which the volume fraction is about $20 \%$ ). It turned out that these hydrodynamic interaction contributions are very small, and are certainly insufficient to explain the experimentally observed distortion in directions perpendicular to the flow direction. The original idea in neglecting the effect of shear flow on shortranged correlation (the fourth step) is as follows. The significance of distortion of short-ranged correlations is measured by the so-called bare Peclet number $P e^{0}$, which is equal to,

$$
P e^{0}=\frac{\dot{\gamma} R_{V}^{2}}{2 D_{0}},
$$

where $D_{0}$ is the Stokes-Einstein diffusion coefficient. From an analysis of the equation of motion for $h(\boldsymbol{r})$ for large distances $r \gg R_{V}$ (see refs. 18 and 19 and below), it follows that the distortion of 
the long-ranged, critical correlations is measured by the dressed Peclet number $\lambda$,

$$
\lambda=\frac{\dot{\gamma} \xi^{2}}{2 D^{\text {eff }}}=\frac{\dot{\gamma} \xi^{4}}{2 D_{0} \beta \Sigma},
$$

where $\xi$ is the correlation length of the quiescent, unsheared suspension, $\beta=1 / k_{\mathrm{B}} T$ and $\Sigma$ is a constant, proportional to the Cahn-Hilliard square gradient coefficient. In the latter equality, which is given here for later reference, we used the following expression for the effective diffusion coefficient at zero wavevector,

$$
D^{\mathrm{eff}}=D_{0} \beta \frac{\mathrm{d} \Pi}{\mathrm{d} \bar{\rho}}
$$

where $\Pi$ is the osmotic pressure of the quiescent, unsheared suspension and $\bar{\rho}=N / V$ is the number density of spheres, and it is used that the correlation length $\xi$ of the unsheared suspension is given by,

$$
\xi=\sqrt{\Sigma / \frac{\mathrm{d} \Pi}{\mathrm{d} \bar{\rho}}} .
$$

Since $\beta \mathrm{d} \Pi / \mathrm{d} \bar{\rho} \rightarrow 0$ on approach of the critical point, the effective diffusion coefficient is much smaller than $D_{0}$ in the neighbourhood of the critical point (which is usually referred to as "critical slowing down"). Furthermore, $\xi \gg R_{V}$ in the vicinity of the critical point. Hence, even when $P e^{0} \ll 1$, the dressed Peclet number $\lambda$ becomes very large on approach of the critical point. This quantifies the intuitive notion that large structures with slow dynamics are much more easily distorted than small structures with fast dynamics. For small bare Peclet numbers, the short-ranged behaviour of the pair-correlation function is simply that of the equilibrium pair-correlation function, in the absence of shear flow. At the same shear-rate, long-ranged, critical correlations are severely affected by the flow. Nevertheless, as will be shown below, distortion of short-ranged correlations are of importance and explain quantitatively the experimentally found distortion of the structure factor in directions perpendicular to the flow direction. What goes wrong in the above reasoning upon close approach of the critical point, is that the leading order term in the equation of motion for $h$ is $\sim \beta \mathrm{d} \Pi / \mathrm{d} \bar{\rho}$, so that terms of order $P e^{0}$ should be compared in magnitude to terms $\sim \beta \mathrm{d} \Pi / \mathrm{d} \bar{\rho}$. Clearly, even for very small bare Peclet numbers, this implies that short-ranged distortions become important on approach of the critical point.

The resulting analytic expression for the static structure factor obtained by invoking the above four steps in solving the $N$-particle Smoluchowski equation ${ }^{18,19}$ is very similar, but not identical, to an expression derived by Ronis ${ }^{21}$ on the basis of a fluctuating diffusion equation.

Instead of fully neglecting the shear-rate dependence of the short-range part of the pair-correlation function, as was done in the theory in ref. 18 and 19, we shall now take into account its leading order distortion, that is,

$$
g(\boldsymbol{r})=g^{\mathrm{eq}}(r)\left[1+P e^{0} g_{1}(\boldsymbol{r})\right], r \leqslant R_{V},
$$

where $g^{\text {eq }}$ is the pair-correlation function of the quiescent, unsheared system. Since for small $P e^{0}$, $g_{1}(r)$ will be only slightly anisotropic, it can be expanded up to second order spherical harmonics as,

$$
g_{1}(\boldsymbol{r})=f_{0}(r)+\hat{\boldsymbol{r}} \cdot \boldsymbol{H}(r) \cdot \hat{\boldsymbol{r}}
$$

where $f_{0}$ is a scalar function and $\boldsymbol{H}$ is matrix, both depending on $r=|\boldsymbol{r}|$. Furthermore, $\hat{\boldsymbol{r}}=\boldsymbol{r} / r$. Without loss of generality, this matrix can be taken traceless and symmetric. For a flow in the $x$-direction with its gradient in the $y$-direction, the matrix elements $H_{13}$ and $H_{23}$ are 0 due to symmetry (where " 1 " is the flow-direction, " 2 " the gradient direction and " 3 " the vorticitydirection). This leaves three independent parameters $H_{11}, H_{22}$ and $H_{12}$. As will turn out, we shall be able to describe our experiments with $H_{11}=0=H_{22}$. Hence,

$$
g(\boldsymbol{r})=g^{\mathrm{eq}}(r)\left[1+P e^{0}\left\{f_{0}(r)+\hat{x} \hat{y} f_{1}(r)\right\}\right], r \leqslant R_{V},
$$


where $f_{1}(r) \equiv H_{12}(r)$. The isotropic distortion $\sim f_{0}$ must be invariant under flow reversal, so that the actual expansion should read,

$$
g(\boldsymbol{r})=g^{\mathrm{eq}}(r)\left[1+\left|P e^{0}\right| f_{0}(r)+P e^{0} \hat{x} \hat{y} f_{1}(r)\right], r \leqslant R_{V} .
$$

The isotropic distortion $\sim f_{0}$ will turn out to be essential to explain the experimentally found distortion of the critical part of the structure factor in directions perpendicular to the flow direction. The form eqn. (8) may be compared to earlier theories on the effect of shear flow on short-ranged correlations. It was Batchelor ${ }^{22}$ who first gave the solution for the pair-correlation function in shear flow for dilute dispersions. Later, this solution was extended to concentrated dispersions by re-scaling ${ }^{23}$ or partially including three-particle correlations, ${ }^{24,25}$ while a linear response analysis for semi-dilute systems is given in ref. 26. In these approximate theories, an isotropic distortion that is linear in the shear-rate is absent. One might thus argue that $f_{0}$ is actually identically equal to zero, and include the isotropic distortion that is $\sim\left(P e^{0}\right)^{2}$. As will be shown in the experimental subsection, however, an isotropic distortion that is linear in the shearrate describes the experimental data significantly better than an isotropic distortion that is quadratic in the shear-rate.

Even for dilute suspensions, the full equation of motion for the pair-correlation function contains integrals over three- and four-particle correlation functions, and fills an entire page. It is not feasible to solve such an equation of motion in full generality. It remains unclear what the origin of the linear, but non-analytic, isotropic contribution in eqn. (8) is. It might be that such a peculiar isotropic contribution is a special feature of near-critical systems. Scattering experiments on nearcritical suspensions that probe large wavevectors might give further, more direct experimental evidence for the validity of eqn. (8).

Repeating the analysis in refs. 18 and 19, but now using eqn. (8) for the short-ranged part of the correlation function, one finds, from the $N$-particle Smoluchowski equation, the following stationary equation of motion for the asymptotic form of the total-correlation function $h(\boldsymbol{r})$ for large distances $r \gg R_{V}$ under stationary shear flow,

$$
0=2 D_{0} \nabla \cdot\left[\left\{\beta \frac{\mathrm{d} \Pi}{\mathrm{d} \bar{\rho}}+\left|P e^{0}\right| \varepsilon^{\prime}\right\} \nabla h(\boldsymbol{r})-P e^{0} \alpha^{\prime} \hat{\boldsymbol{E}} \cdot \nabla h(\boldsymbol{r})-\Sigma \nabla \nabla^{2} h(\boldsymbol{r})\right]-\nabla \cdot[\boldsymbol{\Gamma} \cdot \boldsymbol{r} h(\boldsymbol{r})],
$$

where $\hat{\boldsymbol{E}}$ is the symmetric part of the velocity gradient tensor (divided by the shear-rate, as indicated by the hat on $\boldsymbol{E}$ ). As before, $\Pi$ is the osmotic pressure of the quiescent, unsheared system and $\Sigma$ is a positive constant proportional to the Cahn-Hilliard square gradient coefficient. The two numbers $\varepsilon^{\prime}$ and $\alpha^{\prime}$ originate from the linear shear-rate dependent contribution in eqn. (8), and are given by,

$$
\begin{aligned}
& \varepsilon^{\prime}=\frac{4 \pi}{3} \beta \bar{\rho} \int_{0}^{\infty} \mathrm{d} r r^{3} \frac{\mathrm{d} V(r)}{\mathrm{d} r} g^{\mathrm{eq}}(r) f_{0}(r), \\
& \alpha^{\prime}=\frac{8 \pi}{15} \beta \bar{\rho} \int_{0}^{\infty} \mathrm{d} r r^{3} \frac{\mathrm{d} V(r)}{\mathrm{d} r} g^{\mathrm{eq}}(r) f_{1}(r),
\end{aligned}
$$

where $V$ is the pair-interaction potential. From eqn. (9) it is now clear why the contribution from short-ranged distortions in eqn. (8) is important, although $P e^{0}$ is a small number. On approach of the critical point $\beta \mathrm{d} \Pi / \mathrm{d} \bar{\rho} \rightarrow 0$, so that the small contribution $P e^{0} \varepsilon^{\prime}$ becomes important, despite its small numerical value.

In the first term in eqn. (9) (the term in the curly brackets), we neglected terms of order $\sim h^{2}$ against the term $\sim h \beta \mathrm{d} \Pi / \mathrm{d} \bar{\rho}$ (the second step mentioned in the beginning of this subsection). This is not allowed anymore on very close approach of the critical point, since there $\beta \mathrm{d} \Pi / \mathrm{d} \bar{\rho}$ becomes very small. The region around the critical point where linearization is allowed will be referred to as the "mean-field region". Very close to the critical point, beyond this mean-field region, non-linear equations of motion must be considered. 
Fourier transformation of the equation of motion (9) yields the following equation of motion for the critical part of the structure factor,

$$
\begin{aligned}
\lambda K_{1} \frac{\partial S(\boldsymbol{K})}{\partial K_{2}}= & K^{2}\left(1+\varepsilon+K^{2}\right) S(\boldsymbol{K}) \\
& -K^{2}\left(1+K^{2}\right) S^{\mathrm{eq}}(K)+\alpha K_{1} K_{2}[S(\boldsymbol{K})-1],
\end{aligned}
$$

with the dimensionless wavevector $\boldsymbol{K}=\boldsymbol{k} \xi$, and $S^{\mathrm{eq}}(K)$ is the structure factor of the system in equilibrium, viz. the Ornstein-Zernike structure factor. Here, we have introduced the dimensionless numbers,

$$
\varepsilon=\frac{|\dot{\gamma}|\left(R_{V} \xi\right)^{2}}{2 D_{0} \Sigma} \varepsilon^{\prime}, \quad \alpha=\frac{\dot{\gamma}\left(R_{V} \xi\right)^{2}}{2 D_{0} \Sigma} \alpha^{\prime}
$$

Contrary to the dressed Peclet number $\lambda$ in eqn. (2), the dimensionless numbers $\varepsilon$ and $\alpha$ result from short-ranged interactions, and thereby destroy universality as it is known for a critical system without shear flow. That is, the behaviour of the critical structure factor under shear flow conditions depends on the details of the short-ranged part of the pair-correlation function, and thereby on the details of the pair-interaction potential, contrary to the Ornstein-Zernike structure factor of the unsheared suspension. The stationary equation of motion (11) can be solved analytically.

Note that in directions perpendicular to the flow direction, where $K_{1}=0$, using that the Ornstein-Zernike structure factor is equal to,

$$
S^{\mathrm{eq}}(K)=\frac{\xi^{2}}{\beta \Sigma} \frac{1}{1+K^{2}}
$$

the above result (11) predicts that,

$$
S(\boldsymbol{K})=\frac{1+K^{2}}{1+\varepsilon+K^{2}} S^{\mathrm{eq}}(K)=\frac{\xi^{2}}{\beta \Sigma} \frac{1}{1+\varepsilon+K^{2}}, \quad K_{1}=0 .
$$

This is only equal to $S^{\mathrm{eq}}$ in the case $\varepsilon=0$, that is, with the neglect of the effect of shear flow on isotropic short-ranged correlations. Hence, the isotropic contribution to short-ranged distortions in eqn. (8) is responsible for a finite distortion in directions perpendicular to the flow direction. This also holds when the isotropic distortion would have been, for example, $\sim\left(P e^{0}\right)^{2}$. As mentioned before, our experiments comply with a distortion $\sim P e^{0}$, rather than $\sim\left(P e^{0}\right)^{2}$.

\subsection{Experimental results}

Data were obtained using a small angle light scattering (SALS) set up with an optical, cylindrical shear cell, where the wavevector dependence of the structure factor in the flow-vorticity $\left[K_{1}, K_{3}\right]$ plane is probed. The system used in this study consists of silica spheres (102 nm diameter) grafted with stearyl alcohol, dissolved in cyclohexane. Polydimethylsiloxane (radius of gyration $=23 \mathrm{~nm}$ ) is added to the solution to induce depletion attractions. This sets the range of the pair-interaction potential equal to $R_{V}=148 \mathrm{~nm}$. Due to the attractive depletion forces between the colloidal spheres, this system undergoes a gas-liquid phase transition. The distance to the critical point can be tuned by gently evaporating or adding solvent to a dispersion with the critical ratio of concentration of colloidal spheres and polymers. Scattering patterns were taken for six different correlation lengths $\xi$ (for the quiescent, unsheared system) between $\xi=300$ and $1450 \mathrm{~nm}$, and with shear-rates in the range of $\dot{\gamma}=0$ to $35 \mathrm{~s}^{-1}$. We shall show here data obtained for a system with a correlation length of $\xi=650 \mathrm{~nm}$ (a more extensive report that contains all sets of data points is in preparation). The correlation length is obtained from the Ornstein-Zernike structure factor in the absence of shear flow (for an example of an experimental Ornstein-Zernike structure factor, see Fig. 6).

Fits were performed on ten cross sections in the $\left[K_{1}, K_{3}\right]$-plane per scattering pattern : five cross sections at fixed values of $K_{1}$ as functions of $K_{3}$, and five at fixed $K_{3}$ as functions of $K_{1}$ (these cross sections are indicated by thick lines in Fig. 6). From symmetry consideration, cross sections of 


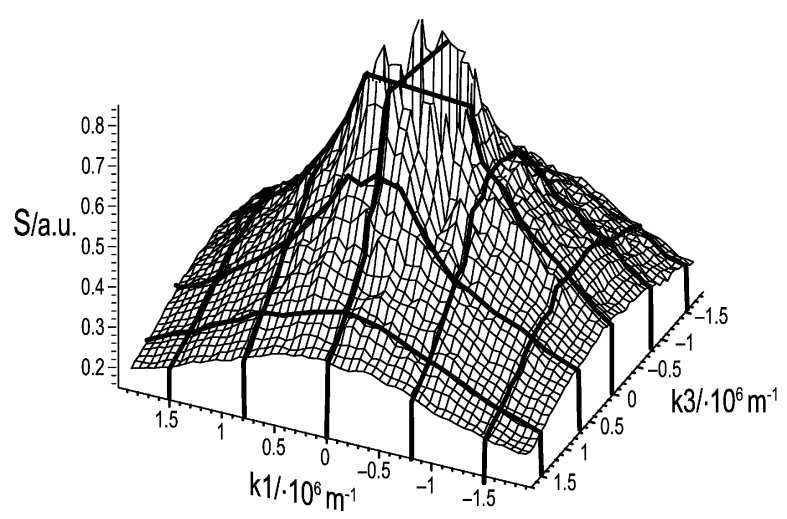

Fig. 6 The Ornstein-Zernike structure factor in the absence of shear flow, for a correlation length of $\xi=650$ $\mathrm{nm}$. The thick, solid lines indicate the cross sections which were used for data analysis.

scattering patterns at opposite sides can be averaged, leading to in total six curves per scattering pattern that were fitted.

Each data set was fitted globally with the equation

$$
\Delta S^{*} \equiv \frac{\beta \Sigma}{\xi^{2}}\left\{S^{\mathrm{eq}}(K)-S(\boldsymbol{K})\right\}=\frac{I^{\mathrm{eq}}(K)-B}{A^{\mathrm{eq}}}-\frac{I(\boldsymbol{K})-B}{A} .
$$

The prefactor $\beta \Sigma / \xi^{2}$ is factored out in the definition of $\Delta S^{*}$, because the solution of eqn. (11) is proportional to $\xi^{2} / \beta \Sigma$, and no absolute intensity measurements have been done. Here, $I^{\mathrm{eq}}(K)$ and $I(\boldsymbol{K})$ are the scattered intensities of the system in equilibrium and under shear, respectively. $A^{\text {eq }}$ and $A$ are the corresponding proportionality constants between the structure factor and the measured intensity, where $A^{\mathrm{eq}}$ is obtained from the Ornstein-Zernike fit of the scattering pattern without shear flow. $B$ is a background that originates from reading noise of the CCD camera and straylight, which is determined from a scattering experiment where the shear cell is filled with solvent. Note that in subtracting the structure factor under shear flow and without shear flow, the non-critical contribution to scattered intensities cancel. Besides the experimental parameter $A$, scattering patterns are fitted with respect to the theoretical parameters $\lambda / \dot{\gamma}$ and $\varepsilon / \dot{\gamma}$. Including the parameter $\alpha$ did not significantly improve fits, so that $\alpha$ is set equal to 0 . It should be emphasized that for a given correlation length, the entire wavevector dependence for a whole set of shear-rates is fitted with just two shear-rate independent theoretical parameters $(\lambda / \dot{\gamma}$ and $\varepsilon / \dot{\gamma})$ and a single experimental, shearrate dependent parameter $(A)$.

Typical least square fitting results are given in Fig. 7 for a correlation length $\xi=650 \mathrm{~nm}$, for two shear-rates. The solid lines are fits to the revised theory (including the term $\sim \varepsilon$ in eqn. (11)), while the dotted lines are fits to the theory where short-ranged correlations are neglected. ${ }^{18,19}$ As can be seen there is a significant distortion in directions where $K_{1}=0$, which becomes more pronounced on closer approach of the critical point (data not shown). This distortion is quantitatively described by the above discussed, revised theory. Fitting without the additional term $\sim \varepsilon$ clearly leads to results that are qualitatively different from the experimental data. The expressions (2) and (12) predict that $\lambda / \dot{\gamma}$ varies like $\sim \xi^{4}$, while $\varepsilon / \dot{\gamma}$ varies like $\xi^{2}$. This is indeed found, as shown in Fig. 8. In the double logarithmic plot in Fig. 8, eqns.(2) and (12) predict slopes of 4 and 2, for $\lambda /$ $\dot{\gamma}$ and $\varepsilon / \dot{\gamma}$, respectively. From a fit we find slopes of $4.4 \pm 0.5$ and $2.2 \pm 0.3$, respectively, in agreement with the predictions in eqns. (2) and (12). Furthermore, from eqn. (2) for $\lambda$ we find a value of $0.78 \pm 0.07$ for the dimensionless group $\beta \Sigma / R_{V}^{2}$, which is of the same order as a crude theoretical estimate of $0.1 .^{18,19,27}$ Furthermore, the value for $\varepsilon^{\prime}$ we find from Fig. 8 is equal to $1.00 \pm 0.08$.

We have also performed least square fits with respect to the shear-rate independent parameters $\lambda / \dot{\gamma}$ and $\varepsilon / \dot{\gamma}^{\nu}$, for various values of the exponent $\nu$. Such fits correspond to expansions of the form eqn. (8), where $\left|P e^{0}\right|$ is replaced by $\left(P e^{0}\right)^{\nu}$. In Fig. 9, the standard deviation of the fits is 

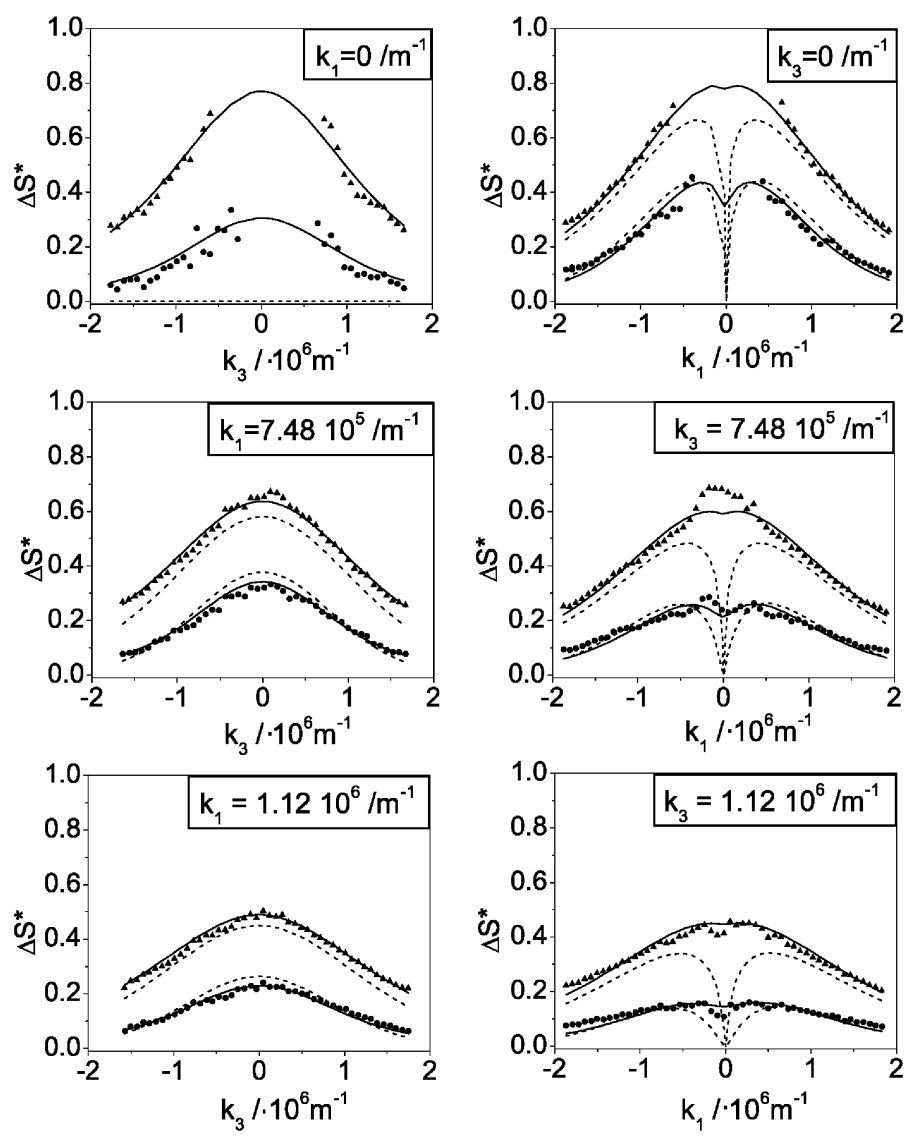

Fig. 7 Typical fitting results for a correlation length of $\xi=650 \mathrm{~nm}$. The points are experimental data, the dotted line is a best least square fit to the theory where distortions of short-ranged correlations are neglected, while the solid lines are best fits to the extended theory. The wavevector at which a cross section is taken is indicated in the right upper corner of each figure.

plotted as a function of $\nu$, for three correlation lengths. Clearly the best fits are obtained for $\nu=1$ $( \pm 0.2)$.

As can be seen from Fig. 7, the extent in the $K_{3}$-direction where there is a significant difference between the two theories complies with a relatively small volume in $\boldsymbol{K}$-space. This explains why integrated quantities, like the shear-viscosity, ${ }^{15}$ turbidity ${ }^{16}$ and shear-induced dichroism ${ }^{17}$ are well described by the older theory in refs. 18 and 19 , more so since the respective integrands are small at small wavevectors. The deviation between experimental dichroism data and the older theory on close approach of the critical point is probably not due to non-mean field effects (as stated in ref. 17 , in the sense discussed above), but is most likely due to the shear-induced short-ranged correlations as described in eqn. (8).

To summarize, we have extended an existing theory on the shear-induced microstructural distortion of near-critical suspensions to include shear-induced distortions of the pair-correlation function at short distances. It is shown that these short-ranged distortions give rise to a significant distortion of long-ranged, critical correlations in directions perpendicular to the flow direction, more so on closer approach of the critical point. The extended theory quantitatively explains small angle light scattering data over a whole range of shear-rates and distances from the gas-liquid critical point. As shown in ref. 28, short-ranged distortions are responsible for the shear-induced shift of the critical point. The finite distortion of critical microstructure in directions perpendicular to the flow direction can therefore be interpreted as being the result of a shear-induced shift of the critical point. 


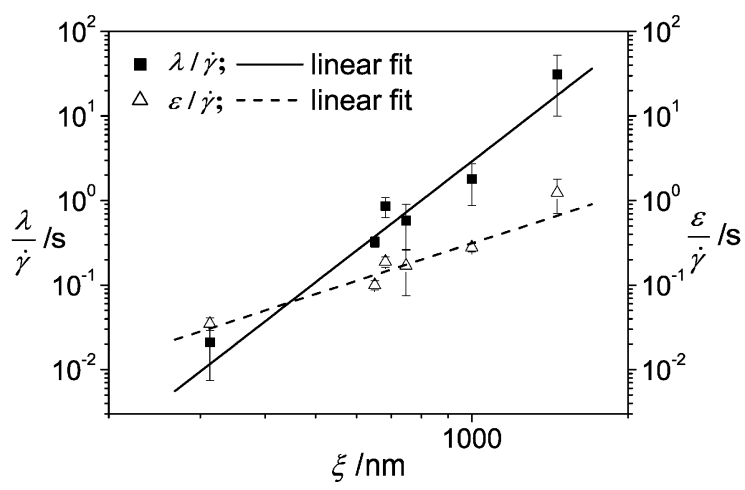

Fig. 8 The best least square fit results for $\lambda / \dot{\gamma}$ and $\varepsilon / \dot{\gamma}$ versus the correlation length $\xi$ of the quiescent, unsheared suspension.

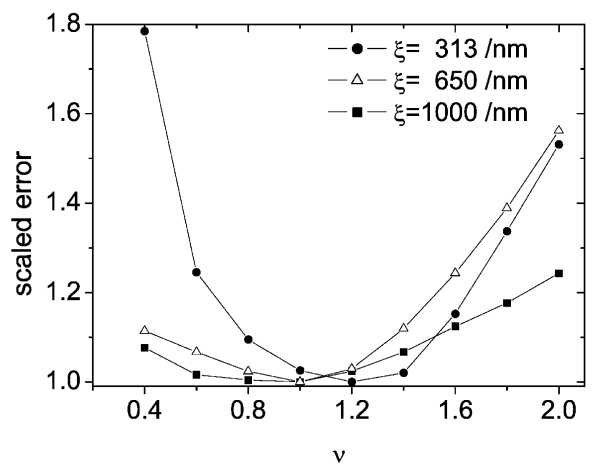

Fig. 9 The minimum standard deviation, from least square fits for various values of the exponent $\nu$. The error here is defined as $\frac{1}{n} \sum_{j}\left(Y_{j}^{\exp }-Y_{j}^{\text {th }}\right)^{2}$, where $n$ is the number of data points involved in the fit, with $Y_{j}^{\exp }$ the $j$-th experimental value for the structure factor and $Y_{j}^{\text {th }}$ the corresponding fitted, theoretical value. Errors are scaled with respect to the minimum standard deviation.

\section{Shear-induced polymer brush deformation}

In this section we shall discuss preliminary neutron scattering and rheology experiments on shearinduced phenomena in suspensions of colloidal particles which are decorated with relatively long polymer chains, so-called "hairy colloids". In particular we shall address shear-induced polymer brush deformation of single, non-interacting hairy spheres. At higher concentrations, interactions between different colloidal particles will have an additional effect on the brush morphology. Brush deformation will influence microstructural order under shear flow conditions in a way that is far from understood yet. In addition, there are indications that such hairy colloidal systems might exhibit shear-banding. ${ }^{29}$

As a model system for hairy colloids we used a poly(ethylene-co-propylene)/poly(ethylene oxide) (PEP-PEO) diblock copolymer micellar suspension in water, where the hydrophilic PEO-block gives rise to the extended polymer brush and the hydrophobic PEP-block forms a dense micellar core (the "Neutron Scattering" group at the IFF in Jülich, led by Prof. D. Richter, studies extensively the equilibrium behaviour of these systems and has long standing experience in their preparation. ${ }^{30}$ There is now a collaborative effort to understand shear-induced form factor and structure factor changes in these systems, and the possible occurrence of shear-banding). With increasing length of the soluble PEO-block relative to the length of the PEP-block that forms the 
core of the micellar structure, there is a transition from a homogeneous sphere with a relatively sharp edge to a more gradual decay of the average density away from the center of the micelle, as seen for star polymers. ${ }^{31}$ Due to the high surface tension of PEP in water, the exchange of chains between different micelles does not occur, even at elevated temperatures. ${ }^{32}$ The advantage of this system is that deuteration of the PEP-block allows one to match the core for neutron scattering. This gives us direct access to the form factor of the deformed PEO-corona. In addition, the ratio of the core radius $R_{\mathrm{c}}$ to the corona thickness $D$ can be varied systematically. The particular diblock copolymer discussed here consists of 370 and 67 PEP and PEO monomers, respectively. This corresponds to an overall molecular weight of $21300 \mathrm{~g} \mathrm{~mol}^{-1}$ and a volume ratio of the two blocks of $V_{\mathrm{PEP}} / V_{\mathrm{PEO}}=0.38$. The core-to-corona size ratio is $R_{\mathrm{c}}: D=1: 1.5$ (see below).

For neutron scattering experiments we used a cylindrical shear-cell (designed by Peter Lindner, ILL, Grenoble), consisting of boron free quartz glass, with a gap width of $1.0 \mathrm{~mm}$. The innercylinder of the shear-cell rotates, it is thermostated by means of a sealed heating envelope with water circulation, and experiments both in the flow-vorticity plane and the gradient-vorticity plane are possible. This shear-cell is integrated in a conventional Bohlin CVO50 stress controlled rheometer.

Neutron scattering experiments were performed at the DIDO reactor of the Reseach Center Jülich, Germany, at a neutron wavelength of $\lambda=0.6 \mathrm{~nm}$ and a bandwidth of $\frac{\Delta \lambda}{\lambda}=0.15$. A $Q$-range of $3 \times 10^{-2} \mathrm{~nm} \leqslant Q \leqslant 1.5 \mathrm{~nm}$ was covered (the wavevector $Q$ was denoted as $k$ in our earlier discussion on light scattering experiments). The direction of observation was along the gradient (radial) direction, so that the flow-vorticity plane is probed. The data were corrected for detector sensitivity, solvent and empty cuvette scattering via standard evaluation procedures. The limited experimental resolution has been taken into account in the fitting routine. The anisotropic, twodimensional SANS data were reduced to one dimension by taking cuts along axes with different orientation relative to the shear flow direction.

Neutron scattering experiments were conducted at a temperature of $20^{\circ} \mathrm{C}$ and a stationary shearrate of $\dot{\gamma}=250 \mathrm{~s}^{-1}$. This was the highest shear-rate before Taylor instabilities interfere.

Small angle neutron scattering (SANS) data are interpreted on the basis of a core-shell model, that has been successfully applied to the corresponding unperturbed system before. ${ }^{30}$ The density profile of a hairy model colloidal particle can be described by the sum of two contributions $n(r)=n_{\mathrm{c}}(r)+n_{\mathrm{m}}(r)$, where $n_{\mathrm{c}}$ and $n_{\mathrm{m}}$ describe the radial distribution in the core region (PEP) and in the corona (PEO), respectively. The density distribution $n_{\mathrm{c}}(r)$ in the core is represented by a convolution of a homogeneous distribution of a sphere of radius $R_{\mathrm{c}}$ and a Gaussian $\exp \left\{-r^{2} / \sigma_{\mathrm{c}}^{2}\right\}$ to account for the smooth transition of the density of the outer part of the core to that of inner part of the brush. The radial decay of the density in the outer shell region is described by a generalized power law dependence,

$$
n_{\mathrm{m}}(r)=r^{-\alpha} \times\left(1+\exp \left\{\left(r-R_{\mathrm{m}}\right) / \sigma_{\mathrm{m}}\right\}\right)^{-1} .
$$

For distances larger than the outer radius $R_{\mathrm{m}}$ of the brush, the Fermi function ensures a fast decay of the density to zero over a distance of about $\sigma_{\mathrm{m}}$. For $\alpha \approx 0$ we obtain a constant density in the shell region while for $\alpha \approx 4 / 3$ the density profile of a star polymer is recovered. ${ }^{33}$ The scattering form factor is obtained by Fourier transformation of $n(r)$. The scattering contribution arising from coherent motion of the PEO-chains that becomes important at larger wavevectors is taken into account as proposed by Dozier and coworkers. ${ }^{34}$ For a visualization of the physical meaning of the model parameters, the density profile of the non-sheared sample, calculated with the parameters $R_{\mathrm{c}}=15.9 \pm 0.1 \mathrm{~nm}, \sigma_{\mathrm{c}}=2.1 \pm 0.1 \mathrm{~nm}, R_{\mathrm{m}}=40.5 \pm 0.4 \mathrm{~nm}, \sigma_{\mathrm{m}} \approx 0 \mathrm{~nm}$, and $\alpha=0.5 \pm 0.1$, as obtained from a least square fit of the SANS data, is plotted in the top part of Fig. 10. The fit turned out not to be very sensitive to the value of $\sigma_{\mathrm{m}}$. Fixing $\sigma_{\mathrm{m}}$ to zero for the sample with and without shear flow did not influence the fit quality. The values for $\sigma_{\mathrm{c}}$ and $\alpha$ did not vary significantly nor systematically around the average values given above and were also kept fixed for the sheared sample. Hence, the only adjustable parameters left for the form factor under shear flow are the core radius $R_{\mathrm{c}}$ and the outer radius $R_{\mathrm{m}}$.

Neutron scattering curves are shown in Fig. 11. Intensities for cross sections along the vorticity (solid symbols) and flow (open symbols) directions are plotted as functions of the scattering vector $Q$. The solid lines are least square fits to the density distribution described above. The deviation at 


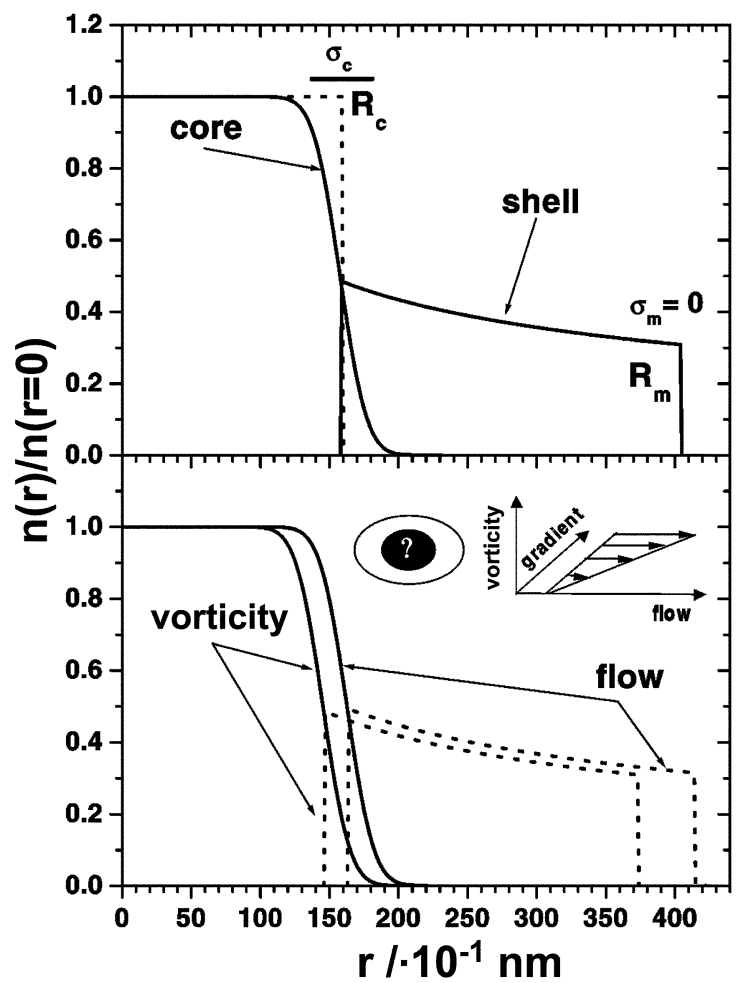

Fig. 10 Upper figure: the density distribution as obtained from neutron scattering data in the absence of shear flow, using the model as described in the main text. Lower figure: resulting density distributions from fits of the neutron scattering data along the vorticity and flow direction at a shear-rate of $250 \mathrm{~s}^{-1}$.

very small $q$-values is due to structure factor distortion effects. The concentration of this sample is 1 wt.\%, which is just large enough to obtain accurate neutron scattering data. Scattered intensities at larger $Q$-values describe the internal structure of a single particle.

In the bottom part of Fig. 10, the density profiles obtained for the sheared sample are shown, both in the flow- and vorticity-direction (which will be indexed as " $\mathrm{f}$ " and " $\mathrm{v}$ ", respectively). The shear-induced deformation of the PEO-brush is clearly visible. We obtain from a fit to eqn. (16) in both directions.

$$
\frac{R_{\mathrm{c}}^{\mathrm{f}}}{R_{\mathrm{c}}^{\mathrm{v}}}=1.11 \pm 0.02 \quad \text { and } \quad \frac{D^{\mathrm{f}}}{D^{\mathrm{v}}}=1.11 \pm 0.02
$$

It thus seems that the PEP-core is just as much deformed as the PEO-brush. These first results suggest that the overall density profile of the sheared sample is prolate-shaped with the longer axis oriented along the flow direction. The observed deformation is of the order of about $11 \%$.

Rheological measurements have been performed in a temperature controlled, double gap configuration, HAAKE RS300 rheometer under controlled stress conditions. Measurements have been made at low concentrations in $\mathrm{D}_{2} \mathrm{O}$. The shear-rate never exceeded about $\dot{\gamma}=10.000 \mathrm{~s}^{-1}$, since above these rates Taylor instabilities occur.

In Fig. 12, normalized shear viscosities for various concentrations are shown. The shear-rate where shear thinning sets in is seen to decrease on increasing concentration. This is due to interactions between different colloidal particles. At higher concentrations the shear viscosity is decreased both due to structure factor distortions and shear-induced brush deformation. In fact, the difference between the scattering curves in Fig. 11 and the form factor (the solid lines) at low values of $q$ is due to the distortion of the structure factor. This kind of distortion is responsible for 


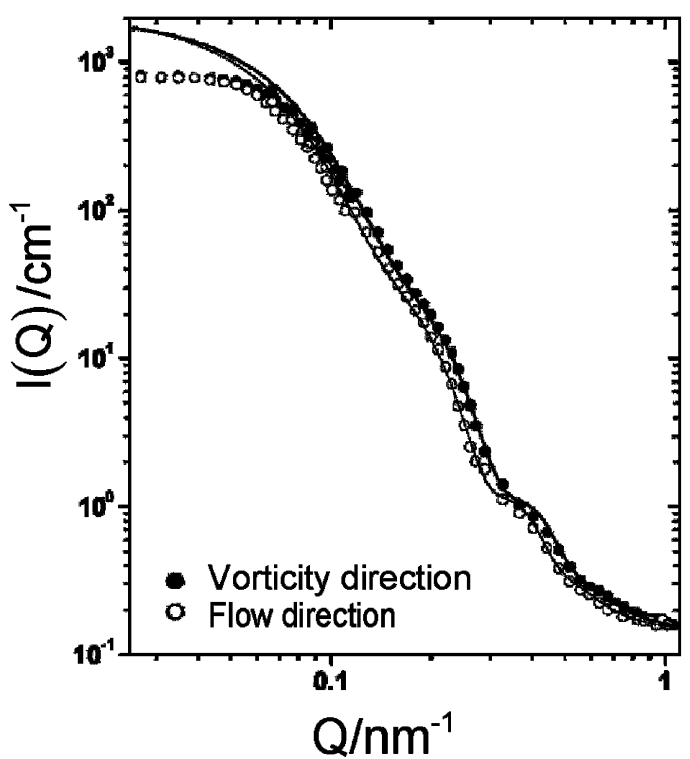

Fig. 11 Neutron scattering curves under shear flow with a shear-rate equal to $250 \mathrm{~s}^{-1}$ in the vorticity direction (solid symbols) and the flow direction (open symbols). The solid lines are fits to the model described in the main text.

the concentration dependence of the shear-rate where shear thinning occurs. It seems that the shear-rate where shear-thinning sets in, extrapolates to a finite value at zero concentration. This finite value connects to polymer brush deformation. At infinite dilution, shear thinning sets in when $\dot{\gamma} \tau \approx 1$, where $\tau$ is the typical time in which a deformed polymer brush relaxes. From Fig. 12 we obtain roughly, $\tau \approx 1 \mathrm{~ms}$.

Larger deformations and resulting shear-thinning at lower shear-rates might result when solvents with a higher viscosity are used. A good- (or theta-) solvent with a much higher viscosity than water will be considered in future research.

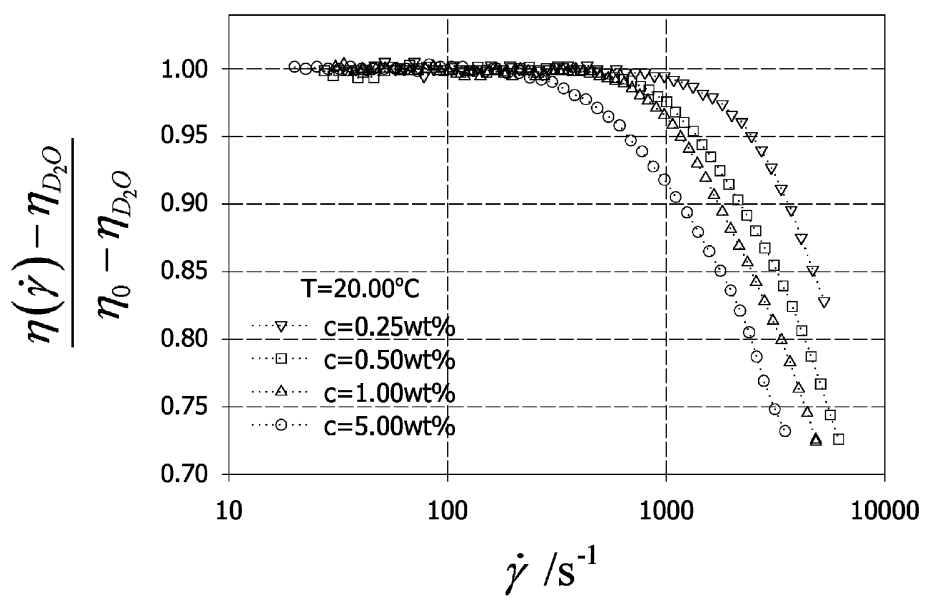

Fig. 12 Viscosity as a function of the shear-rate for different concentrations in wt.\%. 


\section{Acknowledgements}

Financial support by ILL and FZ-Jülich for the development of a specialized SANS/rheology shear cell is gratefully acknowledged. ZD thanks the Humboldt Stiftung for financial support. PC is grateful for financial support from the EC (project "Hard to Ultra Soft Colloids"). Prof. J. Vermant is thanked for his help and discussions concerning the tumbling experiments.

\section{References}

P. Boltenhagen, Y. Hu, E. F. Matthys and D. J. Pine, Phys. Rev. Lett., 1997, 79, 2359.

E. Fischer and P. T. Callaghan, Phys. Rev. E, 2001, 64, 011501.

G. Schmidt, S. Müller, C. Schmidt and W. Richtering, Rheol. Acta, 1999, 38, 486.

4 E. K. Wheeler, P. Fischer and G. G. Fuller, J. Non Newtonian Mech., 1998, 75, 193.

5 J.-F. Berret, D. C. Roux and P. Lindner, Eur. Phys. J. B, 1995, 5, 67.

6 M. E. Cates, Macromolecules, 1987, 20, 2289.

7 J. Tang and S. Fraden, Phys. Rev. Lett., 1993, 21, 3509.

8 T. A. J. Lenstra, Z. Dogic and J. K. G. Dhont, J. Chem. Phys., 2001, 114, 10151.

9 Z. Dogic and S. Fraden, Philos. Trans. R. Soc. London Ser. A, 2001, 359, 997.

10 G. Kiss, J. Polym. Sci., Part B: Polym. Phys., 1996, 34, 2263.

11 R. G. Larson, J. Polym. Sci., Part B: Polym. Phys., 1996, 34, 2267.

12 P. D. Olmsted and P. M. Goldbart, Phys. Rev. A, 1992, 46, 4966.

13 P. D. Olmsted, C.-Y. D. Lu, Phys. Rev. E, 1997, 56, 55; P. D. Olmsted and C.-Y. D. Lu, Phys. Rev. E, 1999, 60, 4397.

14 J. K. G. Dhont and I. Bodnár, Phys. Rev. E, 1998, 58, 4783.

15 I. Bodnár and J. K. G. Dhont, Phys. Rev. Lett., 1996, 77, 5304.

16 H. Verduin and J. K. G. Dhont, Phys. Rev. E, 1995, 52, 1811.

17 T. A. J. Lenstra and J. K. G. Dhont, Phys. Rev. E, 2001, 63, 61401.

18 J. K. G. Dhont, An Introduction to Dynamics of Colloids, Elsevier, Amsterdam, 1996.

19 J. K. G. Dhont and G. Nägele, Phys. Rev. E, 1998, 58, 7710.

20 M. Fixman, J. Chem. Phys., 1960, 33, 1357.

21 D. Ronis, Phys. Rev. A, 1984, 29, 1453.

22 G. K. Batchelor, J. Fluid Mech., 1977, 83, 97.

23 J. F. Brady, J. Chem. Phys., 1993, 99, 567.

24 N. J. Wagner and W. B. Russel, Physica A, 1988, 155, 475.

25 R. A. Lionberger and W. B. Russel, J. Chem. Phys., 1997, 106, 402.

26 G. Szamel, J. Chem. Phys., 2001, 114, 8708.

27 M. Fixman, Adv. Chem. Phys., 1964, 6, 715.

28 J. K. G. Dhont, Phys. Rev. Lett., 1996, 22, 4269.

29 J. Vermant, L. Raynaud, J. Mewis, B. Ernst and G. G. Fuller, J. Colloid Interface Sci., 1999, $211,221$.

30 J. Allgaier, A. Poppe, L. Willner and D. Richter, Macromolecules, 1997, 30, 1582.

31 L. Willner, A. Poppe, J. Allgaier, M. Monkenbusch, P. Lindner and D. Richter, Europhys. Lett., 2000, 51, 628.

32 L. Willner, A. Poppe, J. Allgaier, M. Monkenbusch and D. Richter, Europhys. Lett., 2001, 55, 667.

33 M. Daoud and J. P. Cotton, J. Phys., 1982, 43, 531.

34 W. D. Dozier, J. S. Huang and L. J. Fetters, Macromolecules, 1991, 24, 2810. 